\title{
SPATIAL CRITICAL POINTS OF SOLUTIONS OF A ONE-DIMENSIONAL NONLINEAR PARABOLIC PROBLEM
}

\author{
LAWRENCE TURYN
}

(Communicated by Walter Littman)

\begin{abstract}
The number of spatial critical points is nonincreasing in time, for positive, analytic solutions of a scalar, nonlinear, parabolic partial differential equation in one space dimension. While proving this, we answer the question: What happens to a critical point which loses simplicity?
\end{abstract}

\section{INTRODUCTION}

In recent years there has been a revival of interest in qualitative properties of solutions of nonlinear parabolic partial differential equations on bounded spatial domains. In some sense, for equations modeling diffusion one expects that as one moves forward in time the complexity of the spatial dependence of solutions should decrease. The first, and most fundamental, of such results is the minimum principle, as in Cannon [3] or Protter and Weinberger [10]. Using the minimum principle, further qualitative results have been proven in one space dimension: Nickel [9], as well as others mentioned in Walter [12], proved that the number of spatial extrema is nonincreasing in time, and Matano [7] considered roughly the same concept defined by his "lap number". These results have been useful in proving that the stable and unstable manifolds of two hyperbolic stationary states must intersect transversally, as in Henry [6] and Angenent [1], as well as for proving that critical points are generically (in time) simple, as in Brunovsky and Fiedler [2]. Also, Ni and Sacks [8] have given additional conditions which assure the decrease in the number of spatial critical points of radially symmetric solutions, for any number of space dimensions.

In this note we prove a result with the conclusion that for positive solutions the number of spatial critical points, in one space dimension, is nonincreasing in time. Along the way we consider a question which naturally follows from Brunovsky and Fiedler [2]: What happens to critical points which lose simplicity?

Received by the editors August 15, 1987 and, in revised form, September 25, 1988.

1980 Mathematics Subject Classification (1985 Revision). Primary 35B05, 35K55.

Research supported in part by an ASEE Summer Faculty Fellowship at N.A.S.A. Lewis Research Center, and a Wright State University Research Incentive Grant. 
Three examples are given. The first shows that a critical point can lose simplicity but remain in existence, fixed in space. The second shows that four critical points can coalesce and then disappear, as time increases. The third shows that the main argument, found in Lemma 2 below, could not generalize to more than one space dimension.

\section{THE PROBLEM}

Consider the parabolic partial differential equation initial-boundary value problem

$$
\begin{aligned}
& u_{t}=f\left(x, t, u, u_{x}, u_{x x}\right), \quad 0<x<1, t>0, \\
& u+A(\bar{x}, t) \partial u / \partial n=0, \quad \bar{x} \in\{0,1\}, t>0, \\
& u=u_{0}(x), \quad 0<x<1, t=0 .
\end{aligned}
$$

Here, subscripts denote partial differentiation, and the derivative outward normal to the interval $[0,1]$ is $(\partial u / \partial n)(\bar{x}, t)=-(-1)^{\bar{x}} u_{x}(\bar{x}, t)$ for $\bar{x} \in$ $\{0,1\}$, which is the boundary of $[0,1]$. Assume

(H0) $f\left(x, t, u, u_{x}, u_{x x}\right)=a(x, t) u_{x x}+F\left(x, t, u, u_{x}\right)$.

Denote $D=(0,1) \times(0, \infty)$. Assume

(H1) $a$ is real analytic and positive on $D$;

(H2) $F=F_{1}\left(x, t, u, u_{x}\right) u_{x}+F_{2}(t, u)$, where $F_{1}$ is real analytic on $D \times \mathbf{R}^{2}$, $F_{2}$ is real analytic on $(0, \infty) \times \mathbf{R}$, and $F$ is nonnegative on $D \times \mathbf{R}^{2}$;

(H3) $A(\bar{x}, t)$ is real analytic and nonnegative for $t \in(0, \infty), \bar{x} \in\{0,1\}$;

(H4) $u_{0}$ is real analytic, nonnegative, and not identically zero on $[0,1]$;

(H5) $u_{0}$ is a Morse function on $[0,1]$, i.e. has only simple critical points, i.e. values of $\bar{x}$ with $\left(\partial u_{0} / \partial x\right)(\bar{x})=0$, and only finitely many of them;

(H6) $u_{0}$ has no critical points on the boundary of $[0,1]$, i.e. $\left(\partial u_{0} / \partial x\right)(\bar{x}) \neq$ 0 for $\bar{x} \in\{0,1\}$.

If $\phi:[0,1] \rightarrow \mathbf{R}$ define $\# \phi(\cdot)$ to be the number of critical points of $\phi$, if finite. For any given $u_{0},[0, T)$ will be an interval of existence of the classical solution $u(x, \cdot)$ of $(2.1)-(2.3)$.

Theorem. Assume $(\mathrm{H} 0)-(\mathrm{H} 6)$ and $u(x, t)$ solves $(2.1)-(2.3)$. Then $\# u(\cdot, t)$ is nonincreasing in $t \in[0, T)$.

Lemma 1. Assume $(\mathrm{H} 0)-(\mathrm{H} 4)$ and $u(x, t)$ solves (2.1)-(2.3). Then

(i) $u(x, t)>0$ for $(x, t) \in(0,1) \times(0, T)$,

(ii) $\partial u / \partial x(\bar{x}, \bar{t}) \neq 0$ for $(\bar{x}, \bar{t}) \in\{0,1\} \times(0, T)$.

Proof. (i) follows from the minimum principle, as in [3, 10]. For (ii), suppose not, i.e. that a critical point appears on the boundary of $(0,1)$ at some time $\bar{t}>0$. Then $(\partial u / \partial x)(\bar{x}, \bar{t})=0$ and (2.2) imply $u(\bar{x}, \bar{t})=0$, hence $u$ has an absolute minimum at $(\bar{x}, \bar{t})$. From the Hopf boundary point lemma [10, p. 170], $(\partial u / \partial n)(\bar{x}, \bar{t})<0$, giving a contradiction.

Lemma 2. Assume (H0)-(H4) and $u(x, t)$ solves $(2.1)-(2.3)$ on $(0,1) \times(0, T)$. If $u_{x}(\bar{x}, \bar{t})=u_{x x}(\bar{x}, \bar{t})=0$ for some $(\bar{x}, \bar{t})$ in $(0,1) \times(0, T)$, then for some 
$\delta>0$ the number of solutions $x$ in $(\bar{x}-\delta, \bar{x}+\delta)$ of $0=u_{x}(x, t)$ remains constant or decreases by a multiple of two for $t$ in $(\bar{t}, \bar{t}+\delta)$ versus $t$ in $(\bar{t}-\delta, \bar{t})$.

Proof. Let $n+1$ be the least positive integer such that $D_{x}^{n+1} u(\bar{x}, \bar{t}) \neq 0$. Note that $n$ exists and is greater than one, by the hypotheses, which imply [5] that $u(x, t)$ is real analytic on $(0,1) \times(0, T)$ and, furthermore, that $u_{x}(\cdot, t)$ is not identically zero on $(0,1)$, for $0<t<T$. Throughout, denote $\alpha=a(\bar{x}, \bar{t})$, $\xi=x-\bar{x}, \eta=t-\bar{t}$. The proof distinguishes the cases $n$ even and $n$ odd and uses the Newton polygon [4] to analyze the Taylor series

$$
0=u_{x}(x, t)=\sum_{j, k} D_{x}^{j} D_{t}^{k} u(\bar{x}, \bar{t}) \xi^{j} \eta^{k} / j ! k ! .
$$

Note $D_{x}^{j+1} u(\bar{x}, \bar{t})=0$ for $0 \leq j<n$. Differentiate (2.1) $n-1$ times with respect to $x$, after each differentiation evaluate at $(\bar{x}, \bar{t})$, and use $(\mathrm{H} 1),(\mathrm{H} 2)$, and $D_{x}^{j+1} u(\bar{x}, \bar{t})=0$ for $0 \leq j<n$ to conclude that $D_{x}^{j+1} D_{t} u(\bar{x}, \bar{t})=0$ for $0 \leq j<n-2$ and $D_{x}^{n-1} D_{t} u(\bar{x}, \bar{t})=\alpha D_{x}^{n+1} u(\bar{x}, \bar{t}) \neq 0$. Let $m=[n / 2]$, the integer part of $n / 2$. Similarly, for $0 \leq k<m$,

$$
D_{x}^{j+1} D_{t}^{k} u(\bar{x}, \bar{t})=0 \text { for } 0 \leq j<n-2 m,
$$

and

$$
D_{x}^{n-2 m+1} D_{t}^{m} u(\bar{x}, \bar{t})=\alpha^{m} D_{x}^{n+1} u(\bar{x}, \bar{t}) \neq 0 .
$$

For all integers $n \geq 1$, define $q_{n}(v)=\sum_{k=0}^{[n / 2]} v^{k} /(n-2 k) ! k$ !, which are related to the heat polynomials of Rosenbloom and Widder [11]. Motivated by [11], one observes the facts that for all $n \geq 2$

$$
(n+1) q_{n+1}(v)-2 v q_{n-1}(v)=q_{n}(v)
$$

and

$$
q_{n+1}^{\prime}(v)=q_{n-1}(v) \text {. }
$$

One can establish that, for all $n \geq 2, q_{n}$ can have only simple zeros. This claim is true for $n=2$. If $N+1$ were the least integer for which the claim is false, say $q_{N+1}\left(v_{0}\right)=q_{N+1}^{\prime}\left(v_{0}\right)=0$. From the definition, $v_{0} \neq 0$. Then (2.4) and (2.5) would imply $q_{N}\left(v_{0}\right)=0$, whence $q_{N}^{\prime}\left(v_{0}\right) \neq 0$ and (2.5) would contradict (2.4) $2 v_{0} q_{N-2}\left(v_{0}\right)=N q_{N}\left(v_{0}\right)-q_{N-1}\left(v_{0}\right)$.

First, suppose $n$ is even. After dividing through by $D_{x}^{n+1} u(\bar{x}, \bar{t}),(2.6)$ takes the form

$$
0=\frac{\xi^{n}}{n !}+\alpha \frac{\xi^{n-2}}{(n-2) !} \cdot \frac{\eta}{1 !}+\cdots+\alpha^{m} \frac{\eta^{m}}{m !}+\text { h.o.t. }
$$

where h.o.t. stands for higher order terms which can be neglected, according to the method of the Newton polygon. The latter shows that all solutions of (2.7) are found by the scaling $\eta=\alpha \xi^{2} v$, since $q_{n}$ can have only simple zeros. Because $\alpha=a(\bar{x}, \bar{t})>0$, there are no solutions of (2.7) for $\eta>0$ but, for 
$\eta<0$ sufficiently small, if there are solutions $\xi$ then there are an even number of such solutions.

Second, suppose $n$ is odd. Let $m+l$ be the least positive integer such that $D_{x} D_{t}^{m+l} u(\bar{x}, \bar{t}) \neq 0$, if such $l$ exists. Note that if $l$ exists it is greater than or equal to one, by the previous inductive calculations. After dividing through by $D_{x}^{n+1} u(\bar{x}, \bar{t}),(2.6)$ takes the form

$$
0=\frac{\xi^{n}}{n !}+\alpha \frac{\xi^{n-2}}{(n-2) !} \cdot \frac{\eta}{1 !}+\cdots+\alpha^{m} \frac{\xi}{1 !} \cdot \frac{\eta^{m}}{m !}+\beta \frac{\eta^{m+l}}{(m+l) !}+\text { h.o.t. }
$$

for some $\beta$, possibly zero. Once again, the method of the Newton polygon and the fact that $q_{n}$ can have only simple zeros justifies this truncation and the result that all solutions of (2.8) are found by the scalings $\eta=\alpha \xi^{2} v$ and, if $\beta \neq 0, \xi=\eta^{l} w$. For the first scaling, as was the case for $n$ even, there is a possible decrease of solutions $\xi$ by an even number as $\eta$ passes through 0 ; but, in addition, there can be a solution locally of the form $\xi=O\left(|\eta|^{l}\right)$. For the second scaling, the number of solutions $\xi$ does not change as $\eta$ passes through 0 .

For both $n$ even and $n$ odd, as $\eta$ passes through 0 the number of solutions $\xi$ will decrease by twice the number of real zeros of $q_{n}$. Based on small $n$ Sturm sequences we conjecture that all [ $n / 2]$ distinct zeros of $q_{n}$ are real.

Proof of the theorem. In general, if $u_{x}(\bar{x}, \bar{t})=0 \neq u_{x x}(\bar{x}, \bar{t})$ then the implicit function theorem implies that locally, near $(x, t)=(\bar{x}, \bar{t})$, the solutions of the equation $0=u_{x}(x, t)$ consist of an analytic curve $x-\bar{x}=c(t-\bar{t})+O\left(|t-\bar{t}|^{2}\right)$ for some constant $c$. Thus, (H6) implies that, for some $\delta>0$, \#u(·,t) is constant for $0 \leq t \leq \delta$. Continuations of these analytic curves for $t \geq \delta$, along with the conclusion from Lemma 1(ii) that no critical points appear on the boundary $\{0,1\}$, show that $\# u(\cdot, t)$ could increase only if, for some $(\bar{x}, \bar{t})$, $u_{x}(\bar{x}, \bar{t})=0=u_{x x}(\bar{x}, \bar{t})$. Lemma 2 shows that loss of simplicity of a critical point cannot lead to increase of $\# u(\cdot, t)$.

Remark. The conclusion of the theorem, without the more detailed conclusions found in Lemma 2, could be obtained by slightly different hypotheses: Weaken the analyticity assumptions of $(\mathrm{H} 1)-(\mathrm{H} 4)$ to mere $C^{1}$ smoothness and substantially strengthen (H2) to include the assumptions $F_{2}(t, u) \equiv 0$ and $\left(\partial F_{1} / \partial x\right)(x, t, u, 0) \equiv 0$. Then, differentiate $(2.1)$ with respect to $x$ and obtain a parabolic partial differential equation, for $v=u_{x}$, for which the result [12, p. 207, Theorem III] on the number of "0-points", along with Lemma 1 (ii), yields the conclusion of present theorem.

Example 1. This example from Derek Westwood shows that one of the more complicated conclusions of the proof of Lemma 2 can occur: $u=3 e^{-t} \sin x+$ $e^{-9 t} \sin 3 x$ solves $u_{t}=u_{x x}, u(0, t)=u(\pi, t)=0, u(x, 0)=3 \sin x+\sin 3 x$. Hypotheses $(\mathrm{H} 0)-(\mathrm{H} 6)$ are all satisfied. There are three spatial critical points, 


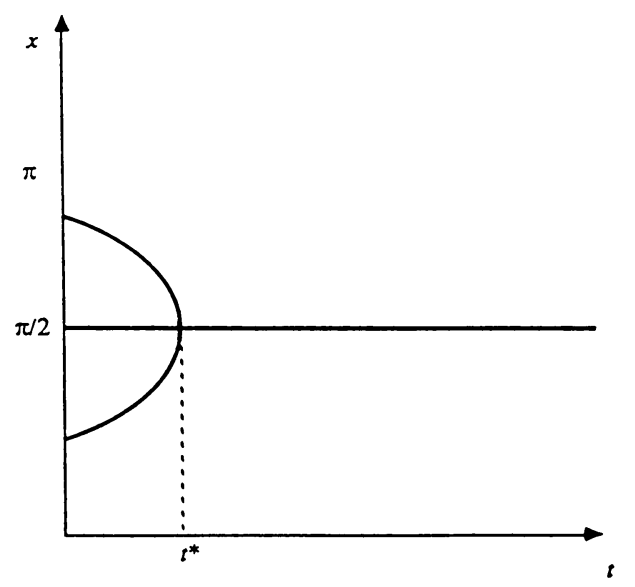

FIGURE 1

including at $x=\pi / 2$, for $0 \leq t<(\ln 3) / 8^{\stackrel{\text { defn }}{=}} t^{*}$, and exactly one critical point, at $x=\pi / 2$, for $t \geq t^{*}$. This example is one of the possibilities of the second case of the proof of the lemma, and the critical points are shown in Figure 1.

Example 2. This example, from the anonymous referee of a previous version of this paper, shows that two other of the conclusions of the proof of Lemma 2 can occur:

$$
\begin{aligned}
u=\mu\left(\frac{\xi^{4}}{4 !}+\frac{\xi^{2}}{2 !} \frac{\eta}{1 !}+\frac{\eta^{2}}{2 !}\right)+\lambda\left(\frac{\xi^{5}}{5 !}+\frac{\xi^{3}}{3 !} \frac{\eta}{1 !}+\frac{\xi^{1}}{1 !} \frac{\eta^{2}}{2 !}\right) \\
\text { solves } u_{t}=u_{x x} \text { for all } \mu, \lambda .
\end{aligned}
$$

For $\mu=0, \lambda=1$, we have the first case of the proof, and the critical points are shown in Figure 2(a). For $\mu=1, \lambda \neq 0$, we have the second case of the proof, and the critical points are shown in Figure 2(b). Note that $u_{x}$ is a linear combination of heat polynomials.

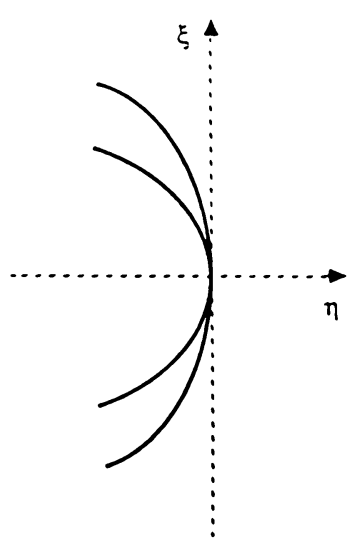

(a)

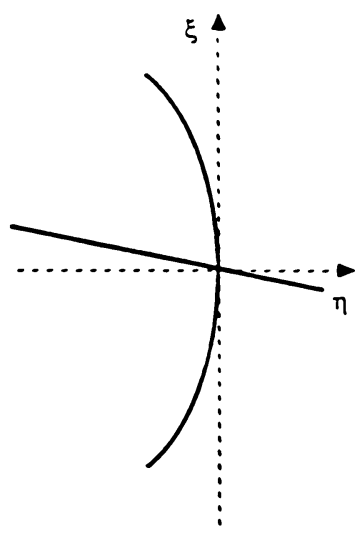

(b)

FIGURE 2 
Example 3. This modification from Nickel [9, p. 93] shows that the Taylor series analysis found in Lemma 2 does not generalize to more than one space dimension: $u=10-2 t-4 x^{2}-2 y^{2}-4_{y}^{4}+2 t y^{2}$ solves $u_{t}=\left[\left(1-y^{2}\right) /(2(3+\right.$ $\left.\left.\left.12 y^{2}-t\right)\right)\right]\left(u_{x x}+u_{y y}\right)$ in the region $\{(x, y)|| x|<1| y \mid,<1\}$. The equation is parabolic for $0<t<3$. There is one critical point, at $(0,0)$, for $0 \leq t<1$, and there are three critical points, at $(0,0)$ and $\left(0, \pm(t-1)^{1 / 2} / 2\right)$, for $1<t<3$. While this example also fails to satisfy (H3), that hypothesis was used in the proof of Lemma 2 (in one space dimension) only to get real-analyticity and nonconstancy of $u$ for $t>0$.

\section{ADDENDUM}

The referee has called our attention to "The dynamics of rotating waves in scalar reaction equations", by S. B. Angenent and B. Fiedler, Trans. Amer. Math. Soc. (to appear). In that paper's $\S 5$, the authors analyze the zeros of solutions $u(\cdot, t)$ of linear heat equations in the same way, using the same tools: Taylor series and the Newton polygon. In fact, our independent work fell short on one detail. The zeros of $q_{n}$ are, besides simple, in fact real, by relating the heat polynomials to the Hermite polynomials. So, the conclusion of our Lemma 2 can be strengthened. Our Lemma 1, on nonexistence of critical points on the boundary of the interval $(0,1)$, is not needed for work on the circle $S^{1}$ by Angenent and Fiedler. Lemma 1 extends to any number of space dimensions, which we intended to work on until we encountered Example 3, due to Nickel.

\section{ACKNOWLEDGMENTS}

Essential to this work was the help of Kenneth R. Meyer, Derek J . Westwood, and the anonymous referee of previous versions of this paper. I would also like to thank Professor Jack K. Hale for suggesting this general subject to me.

\section{REFERENCES}

1. S. B. Angenent, The Morse-Smale property for a semi-linear parabolic equation, J. Differential Equations 62 (1986), 427-442.

2. P. Brunovsky and B. Fiedler, Simplicity of zeros in scalar parabolic equations, J. Differential Equations 62 (1986), 237-241.

3. J. R. Cannon, The one-dimensional heat equation, Addison-Wesley, Menlo Park, California, 1984.

4. S.-N. Chow and J. K. Hale, Methods of bifurcation theory, Springer-Verlag, New York, 1982.

5. A. Friedman, On the regularity of the solutions of nonlinear elliptic and parabolic systems of partial differential equations, J. Math. Mech. 7 (1958), 43-59.

6. D. Henry, Some infinite dimensional Morse-Smale systems defined by parabolic partial differential equations, J. Differential Equations 59 (1985), 165-205.

7. H. Matano, Nonincrease of the lap-number of a solution for a one-dimensional semilinear parabolic equation, J. Fac. Sci. Univ. Tokyo Sect. IA Math. 29 (1982), 401-441.

8. W-M. Ni and P. Sacks, The number of peaks of positive solutions of semilinear parabolic equations, SIAM J. Math. Anal. 16 (1985), 460-471. 
9. K. Nickel, Gestaltaussagen über Lösungen parabolischer Differentialgleichungen, J. Reine Angew. Math. 211 (1962), 78-94.

10. M. H. Protter and H. F. Weinberger, Maximum principles in differential equations, PrenticeHall, Englewood Cliffs, N.J., 1967.

11. P. C. Rosenbloom and D. V. Widder, Expansions in terms of heat polynomials and associated functions, Trans. Amer. Math. Soc. 92 (1959), 220-266.

12. W. Walter, Differential and integral inequalities, Springer-Verlag, New York, (1970).

Department of Mathematics and Statistics, Wright State University, Dayton, Ohio 45435 